\title{
Medulloblastoma in Pregnancy, Case Report from Latifa Hospital, DHA, Dubai, UAE
}

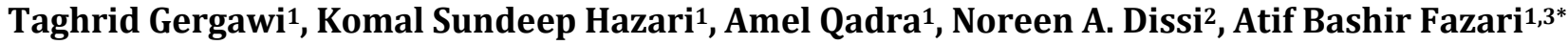 \\ ${ }^{1}$ Department of Obstetrics and Gynecology, Latifa Hospital, DHA, Dubai, UAE \\ ${ }^{2}$ Department of Histopathology, Dubai Hospital, DHA, Dubai, UAE \\ ${ }^{3}$ Faculty of Medicine, University of Medical Sciences \& Technology, Khartoum, Sudan \\ Email: *atiffazari@hotmail.co.uk
}

How to cite this paper: Gergawi, T., Hazari, K.S., Qadra, A., Dissi, N.A. and Fazari, A.B. (2019) Medulloblastoma in Pregnancy, Case Report from Latifa Hospital, DHA, Dubai, UAE. Open Journal of Obstetrics and Gynecology, 9, 129-135.

https://doi.org/10.4236/ojog.2019.92013

Received: January 10, 2019

Accepted: January 30, 2019

Published: February 2, 2019

Copyright $\odot 2019$ by author(s) and Scientific Research Publishing Inc. This work is licensed under the Creative Commons Attribution International License (CC BY 4.0).

http://creativecommons.org/licenses/by/4.0/

\begin{abstract}
Medulloblastoma is a rare brain malignant lesion, reported more in children with less prevalence among adults and rarely seen during pregnancy. The diagnosis of Medulloblastoma is a challenging clinical situation in pregnancy as surgery; radiotherapy and chemotherapy are the choices of management.
\end{abstract}

\section{Keywords}

Medulloblastoma, Pregnancy, Caesarean Section, Craniotomy

\section{Introduction}

Medulloblastoma is an embryonic tumor more prevalent in children than adults [1]. The tumor is highly aggressive but it is sensitive to radiation and chemotherapy [2]. Early diagnosis and surgical removal of the lesion is the key for its management.

Medulloblastomas are the most common malignant brain tumor of childhood and occur exclusively in the cerebellum. Medulloblastoma accounts for about $20 \%$ of the primary CNS tumors with a peak incidence between $5-9$ years and $70 \%$ of them presenting before the age of 20 years. A slight surge may seen during 20 - 24 age but it is rare after 40 years of age [1].

Males displayed higher incidence rates relative to females (males: 0.16 vs. females: 0.12 ), except in patients $<1$ year-old [3].

The signs and symptoms at presentation are due to raised intracranial pressure, cerebellar dysfunction and progression over few weeks to months.

MRI usually shows a contrast enhancing midline or paramedian cerebellar mass; about $30 \%$ of them will have disseminated tumor through subarachnoid space at presentation. 
Medulloblastoma rarely metastasize outside of the nervous system at diagnosis, and therefore systemic staging is not required at the time of diagnosis. The World Health Organization classification of brain tumors divides Medulloblastoma based upon histopathologic criteria into several variants, including classic, desmoplastic/nodular, desmoplastic with extensive nodularity, large cell, or anaplastic. Patients with large cell or anaplastic variants have a significantly poor prognosis. The main stay of therapy involves measures to reduce the raised intracranial pressure and specific tumor guided therapy-utilizing surgery, radiation therapy to the tumor area and craniospinal axis plus systemic chemotherapy [4].

We report this case to identify the clinical presentations, investigation and management of Medulloblastoma during pregnancy at Latifa Hospital, DHA, Dubai, UAE.

\section{Case Report}

A 29 years old primigravida presented at 13 weeks of gestation with history of vomiting and giddiness since the past two months. She had no significant past medical history of concern

On examination, the patient was fully conscious and oriented. The vital signs were reported as follows: Blood pressure: 105/52 $\mathrm{mmHg}$, Pulse rate: 95 beats/minute, Respiratory rate: 14 per minute, Temperature: $36^{\circ} \mathrm{C}$ and the Oxygen saturation: $95 \%$ on room air. There was no pallor or jaundice and she was dehydrated. Systemic review revealed normal findings. Routine investigations and organ function tests as per protocol for vomiting in early pregnancy were done. She received intravenous fluids, Potassium replacement with antiemetic and intravenous vitamin B complex. An obstetric scan was done which revealed a single viable fetus and normal placenta. She showed improvement in her symptoms and biochemistry, and was discharged with antenatal care follow up.

After 3 weeks she reported with projectile vomiting and headache with remarkable nausea over the previous week. Her general condition and nutritional status were good. She was positive for urinary acetone.

She had stable vital signs but was dehydrated. Her lab parameters including blood sugars, electrolytes and thyroid function was within normal range. She was managed with IV fluids, anti-emetics and IV Vitamin B complex. Wernicke's encephalopathy as one of the differential diagnosis was discussed. In spite of symptomatic treatment she went in deterioration of her general condition in form of persistent vomiting and obvious deteriorating neurological status. Direct neurological consultation was performed. She was assessed by the neuro-medical doctor and was given the provisional diagnosis of cerebral venous thrombosis (CVT) with a differential diagnosis of Encephalopathy, side effect of metoclopramide and Conversion disorder (Table 1).

MRI was done and followed by a multidisciplinary team decision including the Neurosurgeon, Obstetrician, and Internal medicine specialist based on the MRI findings, the neurosurgeon decided to operate immediately. The patient 
Table 1. Shows neurological assessment follow up.

\begin{tabular}{|c|c|c|}
\hline & Day 1 & Day 2 \\
\hline Mental status & $\begin{array}{l}\text { Conscious, intermittent } \\
\text { episodes of confusion }\end{array}$ & Confused, disoriented \\
\hline Speech & Clear & Not talking \\
\hline Cranial nerves & $\begin{array}{l}\text { Nystagmus in both the eyes } \\
\text { on looking to the left side }\end{array}$ & $\begin{array}{l}\text { Nystagmus in both the eyes on } \\
\text { looking to the left side }\end{array}$ \\
\hline Ocular movements & Normal & Normal \\
\hline Pupils & Normal size and reactive to light & Normal size and reactive to light \\
\hline Other cranial nerves & $\begin{array}{l}\text { Difficult to assess as the patient } \\
\text { was not following Instructions }\end{array}$ & $\begin{array}{l}\text { Difficult to assess as the patient } \\
\text { was not following Instructions }\end{array}$ \\
\hline $\begin{array}{c}\text { Signs of } \\
\text { meningeal irritation }\end{array}$ & No & No \\
\hline Posture & Stood with support. & Not able to stand \\
\hline Gait & Ataxic gait & Could not be assessed \\
\hline Power & Could not be assessed & Could not be assessed \\
\hline Sensation & Could not be assessed & Could not be assessed \\
\hline Reflexes & Normal. & Normal \\
\hline GCS & $\begin{array}{c}\text { E4V4M5. (E: Eye Opening; } \\
\text { V: Best Verbal Response; } \\
\text { M: Best Motor Response) Score } 13\end{array}$ & $\begin{array}{l}\text { E4V1M5 } \\
\text { Score } 10\end{array}$ \\
\hline
\end{tabular}

and her family were involved in the decision-making in depth counseling with details of the surgery and the risks were explained to them (Figure 1 and Figure 2). A right posterior fossa craniotomy with where total resection of the mass was done at 17 weeks of pregnancy. She tolerated the surgery well without intraoperative or post-operative complications and spent smooth post-operative period under high intensive care.

The histopathology confirmed the diagnosis of Medulloblastoma. The report revealed classic Medulloblastoma composed of densely packed undifferentiated embryonal cells with mild to moderate pleomorphic hyperchromatic nuclei and scanty cytoplasm. Some tumor cells displayed slightly vesicular nuclei with prominent nucleoli. No anaplastic features were seen (Figure 3).

The decision for chemotherapy and radiation was taken after proper counseling with the case and the family to complete the treatment plan and to come out with good prognosis as the tumor well responds to this modality and meanwhile she could continue with the pregnancy for reasonable maturity up to 32 weeks of gestation under close monitoring and follow up in antenatal care clinic. She had been well prepared clinically and psychologically for the preterm delivery, she was counseled well and received steroids. She preferred to travel abroad for elective Caesarean section and to finish with radiation and chemotherapy. The baby was born as preterm and catched up without morbidity with good development. 


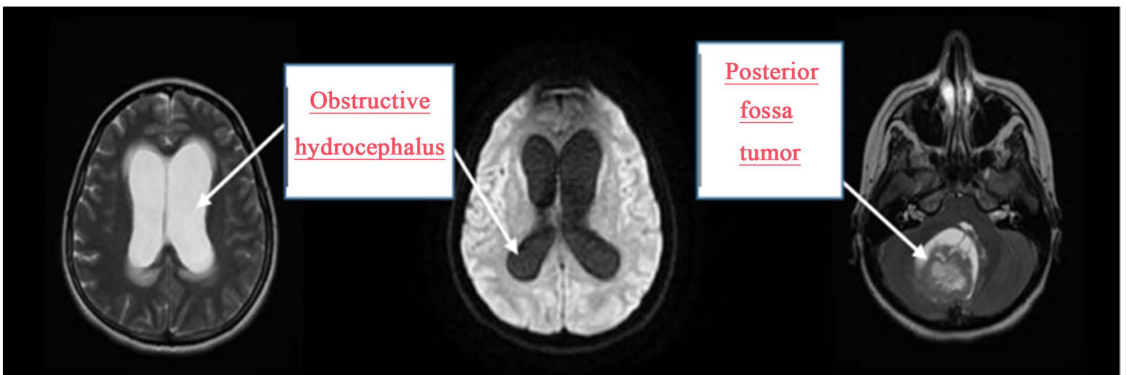

Figure 1. MRI shows intra-axial cerebellar lesion $(4.9 \times 4.3 \times 4.0 \mathrm{~cm})$ involves the right cerebellar hemisphere crosses the midline.

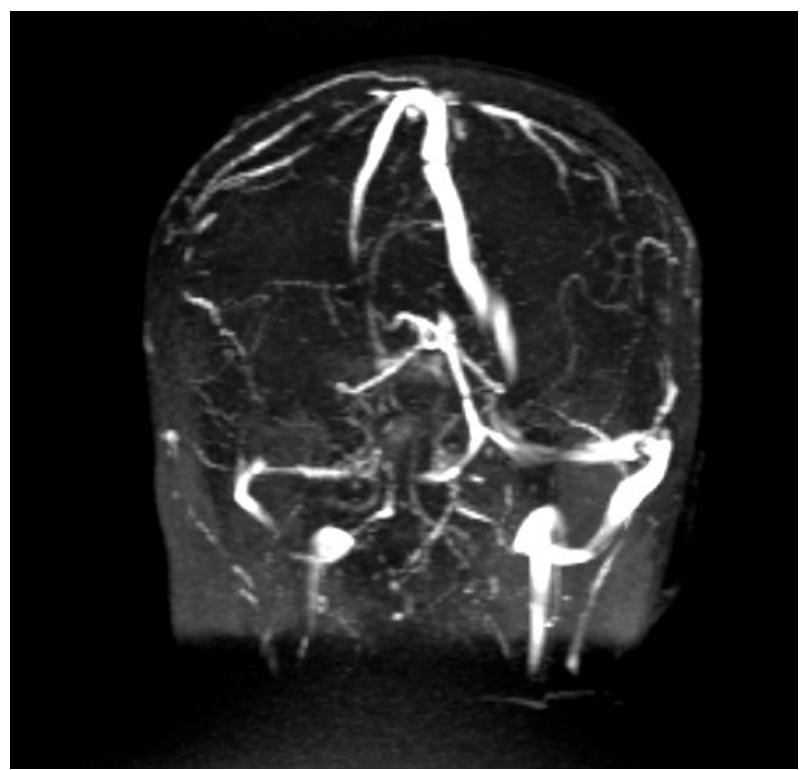

Figure 2. MRV shows normal appearing superior sagittal sinuses, bilateral transverse and sigmoid sinuses. Bilateral internal cerebral veins and vein of Galen appear within normal limits. Right transverse and sigmoid sinuses are relatively small compared to left side; possibly represent normal variation rather than compression.

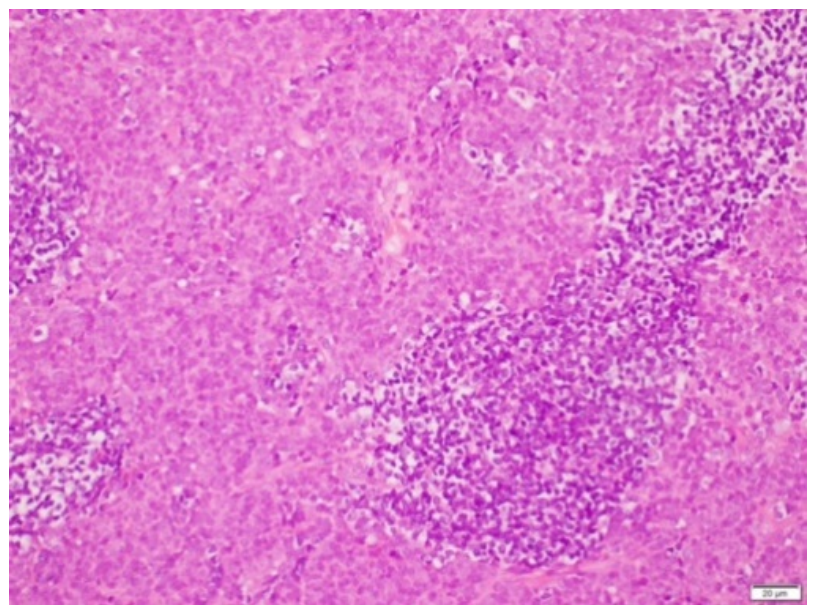

Figure 3. The histopathology of Medulloblastoma composed of densely packed undifferentiated embryonal cells with mild to moderate pleomorphic hyperchromatic nuclei and scanty cytoplasm. 
She tolerated the chemotherapy with some pancytopenia and gastrointestinal symptoms. The agents used were Cisplatin, CCNU (Lomustine) and Vincristine. Local Radiation therapy was done at the site of excised tumor.

She had regular follow up over two years every 3 months with neuro-imaging no signs of relapse or residual tumor. She is stable with no complaint and normal MRI (Figure 4).

\section{Discussion}

Medulloblastomas have an embryonal origin, etiology and pathogenesis is not completely understood. It develops from immature or primitive cells [1].

In the present case, the patient had a prior history of vomiting in pregnancy, treated adequately and discharged. She returned with headache, nausea and projectile vomiting. It was also observed that the symptoms of Medulloblastoma were masked by symptoms mimicking Wernicke's encephalopathy. This misled us to think of other diagnosis like hyperemesis gravidarum, Wernicke's encephalopathy, conversion disorder. The similarity in the symptoms of medulloblastoma with other posterior fossa lesions can cause delayed diagnosis. Thus diagnostic aids like CT and MRI can be helpful in establishing the diagnosis [5].

In the present case, MRI and MRV revealed obstructive hydrocephalous with periventricular seepage of CSF. In addition, a large heterogeneous mass lesion with surrounding small cystic component in the right cerebellar hemisphere causing obstruction of the fourth ventricle was revealed on examination; indicative of a malignant lesion like Medulloblastoma.

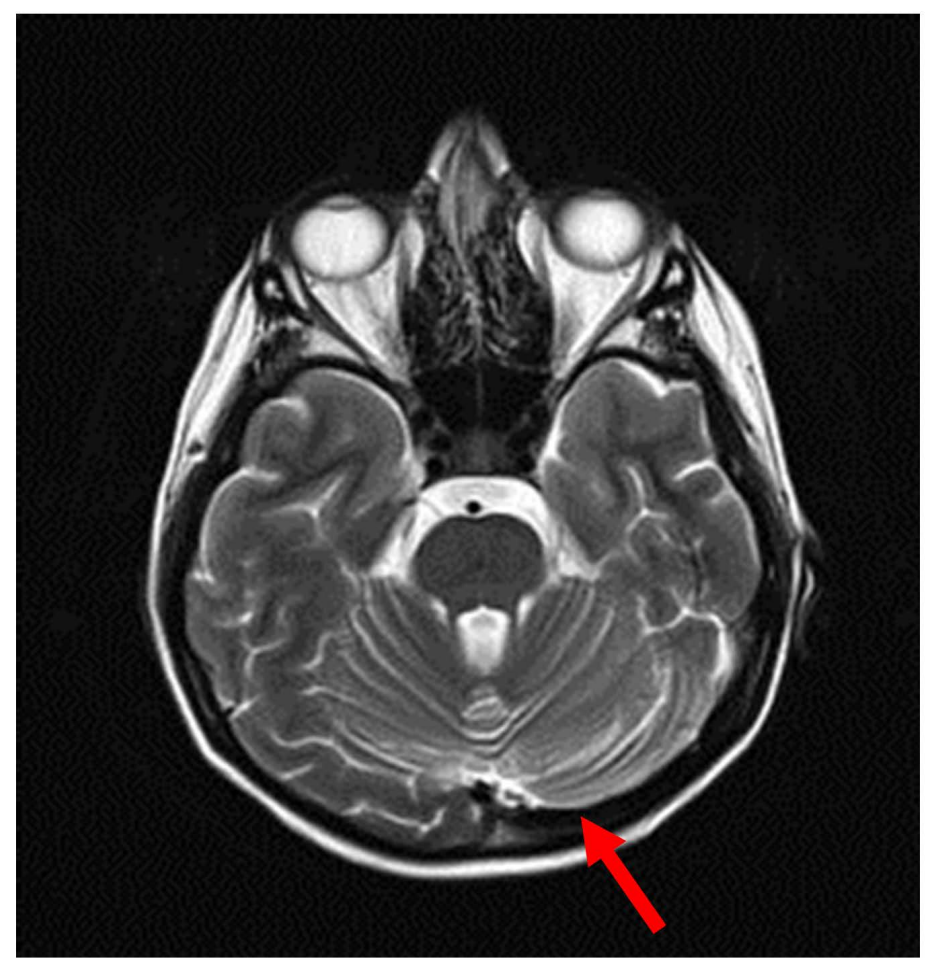

Figure 4. Shows the site of operation (Red arrow). 
Macroscopically, Medulloblastoma appears soft, fleshy greyish tumor accompanied with hemorrhagic areas and small foci of necrosis. Infiltration of the meninges and subarachnoid space along with invasion to the adjoining structures is commonly observed. Microscopically, Medulloblastoma are composed of densely packed cells with round-oval hyperchromatic nuclei and scanty cytoplasm arranged in a diffuse growth pattern. Mild to moderate nuclear pleomorphism may be seen. Homer wright (neuroblastic) rosettes are observed in less than 50\% of cases [6]. Microscopic evaluation of the lesion is the mainstay for establishing a confirmative diagnosis [7]. Immunohistochemistry can be used to confirm the diagnosis. Various markers like Synaptophysin, neuron-specific enolase, MAP-2, and class-III beta tubulin are found to be immunoreactive in cases of medulloblastomas [8]. There are established histologically defined Medulloblasoma variants (classic, nodular/desmoplastic, medulloblastoma with extensive nodularity and large cell/anaplastic variant). This case shows feature of typical classic tumor. It is now widely accepted that there are four genetic (molecular) defined groups of medulloblastoma-WNT-activated (Wingless signaling pathway-activated group), SHH-activated (the Sonic Hedgehog signaling pathway-activated group) and the numerically designated group 3 and group 4 . Both the histologic and molecular subgroups have prognostic and therapeutic value [9].

Once the diagnosis of Medulloblastoma is confirmed the tumor mass should be removed surgically as soon as possible. At times the tumor grows involving the brainstem making its total removal difficult. In such cases the surgery helps to remove as much lesion as possible. This is followed by craniospinal irradiation and chemotherapy [10]. A regular follow up MRI should be done to assess the early evidence of recurrence.

Termination of pregnancy after adequate preparation for the preterm delivery is crucial in case management which was done successfully aiming timed induction of chemo-radiation therapy which is the curable treatment for Medulloblastoma.

Close post treatment follows up and evaluation is part of the care journey to the curable stage.

\section{Conclusion}

Medulloblastoma is an embryonic tumor of childhood with less prevalence in adult hood and even lesser in females. Imaging is the modality of diagnosis. Microscopic evaluation of the lesion is the mainstay for establishing a confirmative diagnosis. Surgery and chemo-radiation therapy is option of treatment. Close post treatment follows up and evaluation is part of the care journey to the curable stage.

\section{Acknowledgements}

Special thanks to Neurosurgery department at Rashid Hospital, DHA, Dubai. Thanks for nurse staff at Latifa hospital and appreciation to Mrs. Kefah Hussni $\mathrm{RN}$ for her input in case care. 


\section{Ethical Approval}

Informed consent obtained from the case herself with agreement of Obstetrics\& Gynecology department for publication

\section{Conflicts of Interest}

Nothing to disclose and conflict of interest.

\section{References}

[1] Smoll, N.R. and Drummond, KJ. (2012) The Incidence of Medulloblastomas and Primitive Neurectodermal Tumours in Adults and Children. Journal of Clinical Neuroscience, 19, 1541-1544. https://doi.org/10.1016/j.jocn.2012.04.009

[2] Akyüz, C., Varan, A., Küpeli, S., Akalan, N., Söylemezoglu, F., Zorlu, F., Kutluk, T. and Büyükpamukçu, M. (2008) Medulloblastoma in Children: A 32-Year Experience from a Single Institution. Journal of Neuro-Oncology, 90, 99-103.

https://doi.org/10.1007/s11060-008-9638-x

[3] Khanna, V., Achey, R.L., Ostrom, Q.T., Block-Beach, H., Kruchko, C., Barnholtz-Sloan, J.S. and de Blank, P.M. (2017) Incidence and Survival Trends for Medulloblastomas in the United States from 2001 to 2013. Journal of Neuro-Oncology, 135, 433-441. https://doi.org/10.1007/s11060-017-2594-6

[4] Pomeroy, S.L., Wen, P.Y. and Gajjar, A. (2014) Clinical Presentation, Diagnosis, and Risk Stratification of Medulloblastoma. Waltham, MA.

[5] Muzumdar, D., Deshpande, A., Kumar, R., Sharma, A., Goel, N., Dange, N., Shah, A. and Goel, A. (2011) Medulloblastoma in Childhood-King Edward Memorial Hospital Surgical Experience and Review: Comparative Analysis of the Case Series of 365 Patients. Journal of Pediatric Neurosciences, 6, 78-85. https://doi.org/10.4103/1817-1745.85717

[6] Massimino, M., Gandola, L., Biassoni, V., Collini, P., Pollo, B. and Giangaspero, F. (2010) Medulloblastoma Histological Variants as the Most Powerful Prognostic Indicator: A 10-Year Mono-Institutional Experience. Journal of Clinical Oncology, 28, 9556.

[7] Sarkar, C., Deb, P. and Sharma, M.C. (2006) Medulloblastomas: New Directions in Risk Stratification. Neurology India, 54, 16-23. https://doi.org/10.4103/0028-3886.24696

[8] Sharma, S. and Tripathi, A. (2016) Pregnant Patient Presenting with Headache and Medulloblastoma and Hydrocephalous: A Case Report. International Journal of Reproduction, Contraception, Obstetrics and Gynecology, 3, 229-230.

[9] Louis, D.N., Perry, A., Reifenberger, G., Von Deimling, A., Figarella-Branger, D., Cavenee, W.K., Ohgaki, H., Wiestler, O.D., Kleihues, P. and Ellison, D.W. (2016) The 2016 World Health Organization Classification of Tumors of the Central Nervous System: A Summary. Acta Neuropathologica, 131, 803-820. https://doi.org/10.1007/s00401-016-1545-1

[10] Fukunaga-Johnson, N., Lee, J.H., Sandler, H.M., Robertson, P., McNeil, E. and Goldwein, J.W. (1998) Patterns of Failure Following Treatment for Medulloblastoma: Is It Necessary to Treat the Entire Posterior Fossa? International Journal of Radiation Oncology Biology Physics, 42, 143-146. https://doi.org/10.1016/S0360-3016(98)00178-3 